\title{
MALLAS TURÍSTICAS. La reinvención de un tejido como soporte de la actividad vacacional en Mallorca*
}

\author{
Biel Horrach Estarellas \\ Universitat Politècnica de Catalunya
}

\section{RESUMEN}

Desde mediados de los años 50, con el nacimiento del turismo de masas en Europa, la isla de Mallorca se convierte en uno de los principales destinos turísticos de sol y playa a nivel mundial. A raíz del importante desarrollo experimentado se genera un complejo sistema de asentamientos, que transforma buena parte del paisaje litoral. La investigación expone una metodología de decodificación transescalar de este territorio mediante el reconocimiento morfológico de los tejidos específicamente turísticos que lo conforman.

\section{ABSTRACT}

In the middle fifties, with the birth of the mass tourism in Europe, Majorca Island became one of the main world tourist sun and beach destinations. A complex system of settlements was generated, transforming the coastline landscape. This article proposes a decoding methodology spanning multiple scale levels via the morphological recognition of settlement-units specifically related to tourism.

\footnotetext{
Dicho artículo se basa en parte de la investigación titulada: "La balearización. Mallorca el laboratorio de experimentación del turismo y su manifestación en el litoral", presentada como tesis del Máster de Investigación en Urbanismo del Departamento de Urbanismo y Ordenación del Territorio de la Universitat Politècnica de Catalunya en septiembre de 2008.
} 


\section{INTRODUCCIÓN}

Si a lo largo del siglo $X X$ el turismo se ha consolidado como una de las principales actividades económicas del planeta, Mallorca ha sido uno de los principales laboratorios mundiales del turismo litoral. Esta isla del Mediterráneo Occidental, de apenas $3.640 \mathrm{Km}^{2}$ y 850.000 habitantes, ha contado con uno de los más importantes aeropuertos turísticos de Europa, superando actualmente los 20 millones de pasajeros al año.

Después de más de 100 años construyendo un complejo sistema de asentamientos turísticos litorales se ha generando la necesidad de dar una respuesta a las nuevas motivaciones cualitativas de la demanda turística actual planteándose especialmente dos cuestiones: ¿Cómo se deben desarrollar los productos turísticos adaptados a la nueva demanda? y ¿qué modelo aplicar a los espacios turísticos asociados a la masividad y estandarización existentes?

El principal objetivo de mi investigación doctoral consiste en reconocer los procesos de transformación territorial que ha generado la actividad turística en el litoral de Mallorca, como herramienta para reconducir algunos de los procesos desarrollados hasta la actualidad. Y lo hace mediante la decodificación de la gran diversidad de formas de asentamientos vacacionales en cuatro tipologías: los asentamientos suburbanos, las mallas turísticas, la ciudad jardín vacacional y los centros autónomos.

El presente artículo se centra en las mallas turísticas, tipología caracterizada por ser la más extendida entre los diferentes desarrollos turísticos, debido especialmente a su alta capacidad de integrar una gran diversidad de productos turísticos, tipologías hoteleras y de apartamentos, así como de segunda residencia.

Después de la evolución de las mallas decimonónicas, desde proyectos de extensión urbana hasta núcleos poblacionales de nueva creación, con la llegada de la actividad turística estos tejidos experimentan un importante

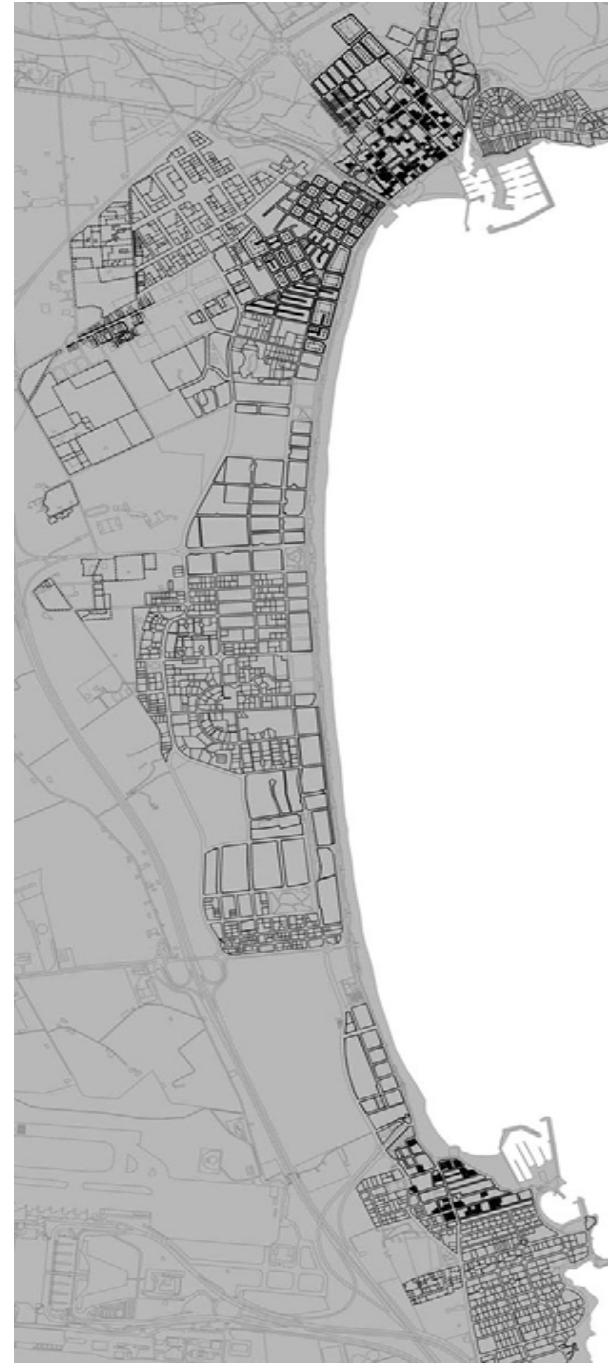

El sistema turístico-territorial de la Playa de Palma. Y las unidades de tejidos turísticos que lo conforman Fuente: elaboración propia 
proceso de transformación que las dota de características específicamente turísticas.

Los principales factores de ubicación y ordenación de las mallas turísticas se han basado en su relación con su principal recurso, el paisaje litoral. El paisaje más valorado ha experimentado una serie de variaciones vinculadas a los cambios de modelo consolidado en cada etapa, así como al imaginario turístico asociado a él. De manera que, a través de la experiencia, la propia actividad turística ha ido reinventando las formas de ordenación de los tejidos en malla hasta obtener los espacios óptimos para pasar el tiempo vacacional.

\section{PAISAJE Y TURISMO, CATALIZADORES DE UN PROCESO}

Todo empezó en el litoral mallorquín a mediados del siglo XIX cuando, después de permanecer siglos prácticamente deshabitado por cuestiones sanitarias y de seguridad, empezó a colonizarse a través de diversos proyectos de núcleos de nueva creación. La mayoría de ellos responde a las "Leyes estatales de Colonias Agrícolas", que se dieron a partir de la segunda mitad del siglo XIX, para impulsar la agricultura, ganadería e incluso, en algunos casos, el comercio marítimo. Los asentamientos resultantes empiezan muy pronto a presentar una serie de características que los singularizan del resto de colonias agrícolas. Pasan a combinar las actividades económicas de origen con el uso vacacional. $Y$ es que muy pronto, muchas de las familias acomodadas de los pueblos del interior empiezan a construir aquí sus primeras residencias de veraneo.

La tipología edificatoria resultante es una evolución de la casa tradicional mallorquina de los pueblos del interior (García-Delgado, 1996) incorporando una serie de estancias que permiten una mayor relación y disfrute de los espacios exteriores. Por ejemplo, la aparición del porche en fachada conforma una nueva sección de la calle en los tejidos en malla. $Y$ así sucesivamente se van diferenciando las características espaciales de las mallas costeras

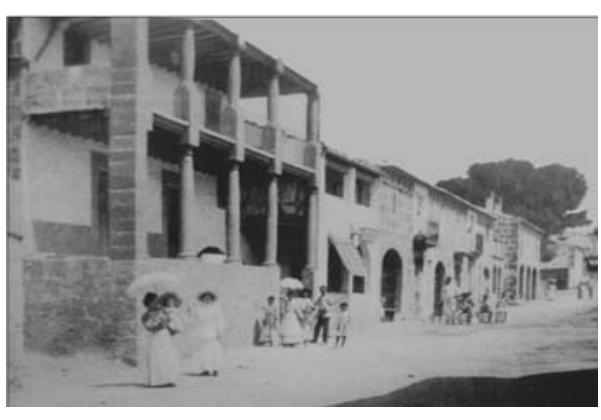

Reinvención de la casa tradicional. Calle Sant Cristòfol de la Playa de Palma a principios del s. XX, con las nuevas tipologías edificatorias de carácter vacacional.

Fuente: foto archivo P. Francisco Salvá.

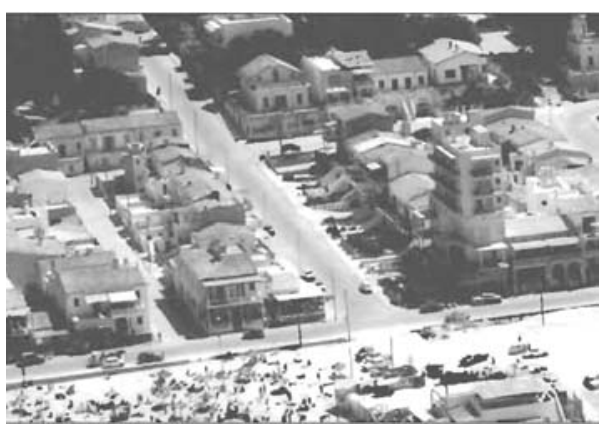

La consolidación de las primeras mallas turísticas. Estructura urbana resultante del Arenal de la Playa de Palma a mediados del s. XX.

Fuente: P.R.I. Playa de Palma 
respecto al resto de colonias agrícolas y de los ensanches tradicionales que se están desarrollando tanto en Palma, como en algunas poblaciones del interior.

Una de las principales aportaciones de las mallas turística es la de conformar los primeros tejidos de soporte del turismo de masas, experimentando los nuevos productos que aparecen en el mercado.

Corren los años 50, y la inversión en nuevas infraestructuras en el espacio turístico son muy escasas. Así que como respuesta al turismo de sol y playa se fueron insertando las primeras instalaciones hoteleras en las mallas litorales existentes. El cambio de tipología edificatoria consistía en la agregación del parcelario y el desarrollo de establecimientos hoteleros en altura que permiten aumentar la capacidad de alojamiento y multiplicar por diez plantas la capacidad de ofrecer vistas al mar, uno de sus principales recursos. La suma de intereses particulares acaba conformando mallas turísticas densificadas que con los años difícilmente pueden responder a las necesidades del producto sol y playa.

Más tarde, con la voluntad de regular el número de plantas edificatorias se aplican ordenanzas más propias de la ciudad. La altura reguladora está en función de la anchura de la calle y el patio interior de manzana, conformando manzanas cerradas de tamaño reducido, de manera que muchas de las mallas turísticas transformadas pierden los valores espaciales originales, sin hacer ninguna aportación al espacio turístico resultante.

Con la reinversión en el espacio turístico, tras la aprobación de la Ley de Zonas y Centros de Interés Turístico Nacional (1963), se desarrollan nuevas extensiones en malla asociadas al turismo de masas. Se pasa de la unidad de alojamiento entre medianeras a la edificación aislada, experimentando un aumento del tamaño de la manzana en función de la tipología de establecimiento turístico, así como de las características paisajísticas de su entorno. Las primeras extensiones en malla tienden a ubicarse en primera línea

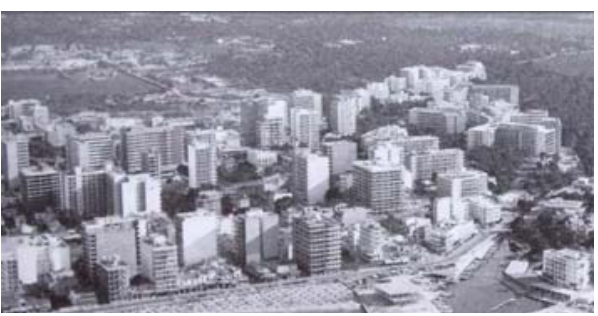

Densificación de las mallas turísticas tradicionales Foto aérea del Arenal de 1980.

Fuente: archivo Pere Canals Morro

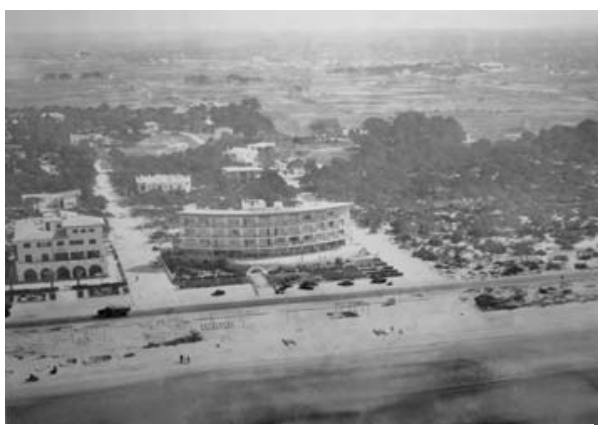

Primeras mallas turísticas modernas Hotel Lido y Hotel Acapulco sobre el sistema dunar de la Playa de Palma en 1964.

Fuente: Hotel Acapulco 
de mar, sobre el sistema dunar, buscando una relación directa con la playa, su principal recurso.

Posteriormente, conscientes de que la primera línea de mar no es ilimitada, se empiezan a hacer macrooperaciones que se ubican entre el frente marítimo y las nuevas vías territoriales dan acceso, ocupando suelo agrícola. La instalación hotelera pierde la relación directa con la playa, e incorpora un espacio libre privado de dimensiones cada vez mayores, para ofrecer al cliente todos los productos sin necesidad de salir del establecimiento.

Las lógicas de ubicación, así como las de ordenación, han venido definidas por el máximo aprovechamiento de los recursos turísticos. Inicialmente, cuando había mayor disponibilidad de emplazamientos, las características paisajísticas del entorno son el principal factor. Hasta los años cincuenta las mallas turísticas suelen mantener una ubicación respetuosa con los elementos que estructuran el territorio. Posteriormente, con la llegada del turismo de masas se produce un detrimento de la calidad paisajística. Los nuevos asentamientos conforman estructuras autistas respecto a su entorno rural, desdibujando el sistema hidrográfico y las marismas, y ocupando el sistema dunar en el frente de la playa.

Cuando desaparece la posibilidad de localizar los tejidos turísticos en los parajes más atractivos, se hace evidente su incapacidad para generar un nuevo espacio público de suficiente entidad, como ha sido la playa y el paseo marítimo. Con la pérdida de confianza en los espacios libres públicos se van incorporando nuevas lógicas de ordenación en que el espacio libre privado pasa a ser su principal recurso. En muchos casos se conforman interesantes formas de agregación por vecindad entre parcelas, permitiendo dar continuidad a pinedas existentes.

Sin embargo, las tendencias cada vez más globalizadas influencian el imaginario turístico, manifestándose en el espacio vacacional con la importación de productos totalmente ajenos a la realidad del territorio

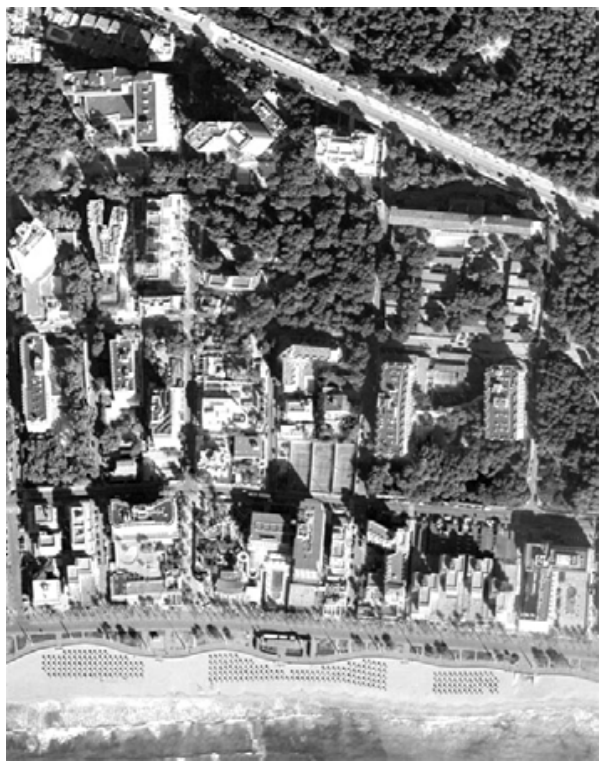

Evolución de la malla turística en relación a la calidad paisajística.

Evolución del espacio libre privado en función del distanciamiento con la playa.

Fuente: IDEIB gobierno Balear. 
mallorquín. Es así como se importan aquellos paisajes que hacen referencia a lugares o actividades considerados por el imaginario turístico como idílicos; unos vinculados a actividades deportivas de elite, como el golf, mientras que otros propios de los nuevos destinos mundiales emergentes, produciéndose la tropicalización del litoral mallorquín. En consecuencia, la pérdida de identidad del espacio turístico mallorquín está dificultando la capacidad de ser competitivo frente a otros destinos, si no es mediante el abaratamiento de las estancias y la consecuente pérdida de valor añadido.

Por otro lado, se ha experimentado una sucesiva pérdida de urbanidad, ocasionada por el aumento del tamaño de la parcela y la mínima relación de la instalación turística con el espacio público, dando como resultado manzanas de casi un kilómetro de perímetro con accesos puntuales a los establecimientos con actividad.

\section{REPENSAR LAS MALLAS TURÍSTICAS}

Las mallas que conforman los tejidos turísticos presentan unas características diferenciales respecto a las que construyen la ciudad, dando respuesta a la vez a las nuevas motivaciones del turista, como son la autenticidad, flexibilidad y diversidad del producto turístico. Por tanto se requieren unas lógicas de ordenación y puesta en valor diferentes. Desde esta lectura se presentan tres claves para la reconversión del espacio turístico a partir de las oportunidades que ofrecen las propias mallas turísticas:

- La potenciación de los valores de cada malla turística en función de sus características morfo-tipológicas. Es decir, potenciando en cada una un recurso turístico diferenciado, en función de su densidad, tipología edificatoria, usos, sistema de espacios libres, y dotando al conjunto de una gran diversidad de productos. Por ejemplo, la malla turística densificada presenta una alta mezcla
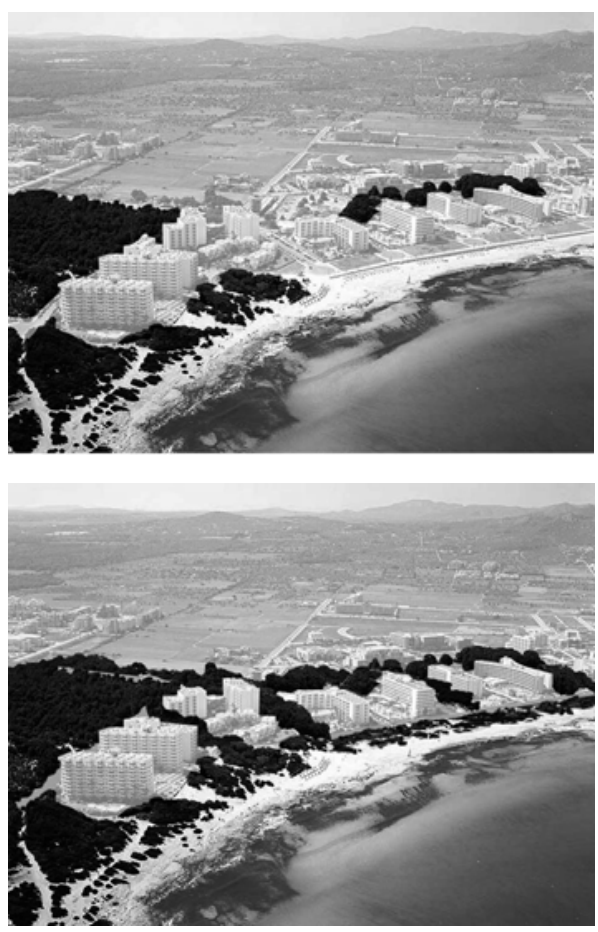

Reconversión de la malla turística moderna. Integración del sistema dunar con la malla turística de Cala Millor.

Fuente: elaboración propia a partir de fotos aéreas del Ministerio de Medio Ambiente. 
de usos capaz de mantener activo el espacio público a través de nuevas actividades que complementan el producto de sol y playa.

- La recuperación de los elementos que estructuran el territorio, potenciando los valores paisajísticos que lo conforman, como pueden ser el sistema dunar y las pinedas litorales, las zonas húmedas y el sistema hidrográfico, así como el paisaje agrario, integrándolo en los límites de las mallas turística. De esta manera, se dota de identidad a dichos tejidos a partir de los propios valores del lugar.

- La integración del espacio libre privado a la estructura de espacios libres colectivos, conformando una estructura de espacios abiertos con actividad, mediante la permeabilización de los jardines y zonas verdes de cada establecimiento turístico al uso colectivo.

En definitiva, la necesidad de comprender el espacio turístico litoral desde una lectura transescalar, más allá de la clásica visión concentrada en la instalación turística (Barba, 1992), se pone en evidencia, para entender no sólo la forma edificatoria resultante sino también el tejido, de características específicamente turísticas, así como los valores del paisaje en la que se encuentra ubicado. Porque en el paisaje propio del lugar está la clave para la puesta en valor de los tejidos turísticos existentes.

\section{BIBLIOGRAFIA}

BARBA, Rosa (1992). "Peces mínimes i turisme". Quaderns d'arquitectura i Urbanisme nº 194. p 44-47. Barcelona.

GARCÍA-DELGADO, Carlos (1996). La casa popular mallorquina. José J. de Olañeta, editor. Palma de Mallorca.

RULLÁN, Onofre (2004). La construcción territorial de Mallorca. Palma de Mallorca.

SEGUÍ LLINÀS, Miguel (2006). El turisme a les Illes Balears (1950-2005). Edicions Documenta Balear. Palma de Mallorca. 